\title{
Hamilton Energy Control for the Chaotic System with Hidden Attractors
}

\author{
Meibo Wang (D) $^{1}$ and Shaojuan Ma (D) $^{1,2}$ \\ ${ }^{1}$ School of Mathematics and Information Science, North Minzu University, Yinchuan 750021, China \\ ${ }^{2}$ The Key Laboratory of Intelligent Information and Big Data Processing of Ningxia Province, Yinchuan 750021, China
}

Correspondence should be addressed to Shaojuan Ma; sjma@nmu.edu.cn

Received 2 March 2021; Accepted 27 July 2021; Published 4 August 2021

Academic Editor: Xinzhi Liu

Copyright (c) 2021 Meibo Wang and Shaojuan Ma. This is an open access article distributed under the Creative Commons Attribution License, which permits unrestricted use, distribution, and reproduction in any medium, provided the original work is properly cited.

\begin{abstract}
In this paper, the dynamic behavior and control of chaotic systems with hidden attractors are studied. Firstly, a class of autonomous chaotic systems without the equilibrium point is proposed. Secondly, quantitative analysis methods are applied to explore the dynamic behavior of the new chaotic systems. Then, the Hamilton energy function of the new system is calculated by the Helmholtz theorem and the energy feedback controller is designed. Finally, the effectiveness of the controller is verified by numerical simulations. Compared with the line feedback control, the control effect of Hamilton energy control is better.
\end{abstract}

\section{Introduction}

Since the second half of the twentieth century, nonlinear science has made great development. Chaos, as a research hotspot of nonlinear science, is considered to be the third revolution after relativity theory and quantum mechanics [1]. So, it is paid more attention by many researchers. They found some important properties about different kinds of chaotic systems, especially including chaotic systems with hidden attractors [2-6]. These attractors are widely present in different dynamic systems, such as the Chua circuit [7], Van der Pol-Duffing oscillator [8], induction motor-driven drilling system model [9], convective fluid motion-like Lorenz system [10], and multilevel DC converter [11]. Hidden attractors are not intersected with any small neighborhoods of unstable equilibrium points, which can lead to unpredictable catastrophic responses, such as airplane crashes, sudden climate changes, severe diseases, financial crises, and commercial equipment problems $[12,13]$. Therefore, the research studies on chaotic control of the systems with hidden attractors are of great significance. Sharma et al. [14] applied the scheme of linear augmentation to control the multistability in the hidden attractors. Feng and Wei [15] studied time-delay feedback control to the generalized Sprott B system with hidden attractors. Adaptive control of the hyperchaotic system with a hidden attractor was designed by Vaidyanathan [16]. Wei et al. [17] applied the nonlinear feedback controller, sliding mode controller, and their hybrid combination to control the chaotic system with hidden attractors. In circuits [18-22], Lai et al. proposed a no-equilibrium chaotic system with hidden attractors and coexisting attractors [22]. Marius and Michal [23] proved that the impulsive difference equation can generate hidden attractors; they also restrained the chaotic behavior of one-dimensional discrete dynamical systems by using pulse control. The construction of multiple hidden attractors was achieved by $\mathrm{Wu}$ et al. [24] through a universal pulse control. In [25], a sliding mode controller was used to control a three-dimensional multistate time-delay chaotic system with hidden attractors.

About the control of chaotic systems with hidden attractors, the traditional control strategies have already achieved the above research studies. Recently, many control methods have been introduced into chaotic systems, such as energy information. The Hamiltonian energy functions of some classical chaotic systems were calculated and verified by Sarasola [26-28]. In 2005, Sarasola et al. [29] proposed chaotic systems with phase-space variable functions and 
analyzed the energy flow under different coupling intensities. Torrealdea et al. [30,31] described the energy function of a Hindmarsh-Rose neuron and evaluated the energy consumption of the neuron during its signal activity. The energy effect on synchronization for a pair of structurally flexible coupled neurons was studied by Moujahid et al. [32]. In [33], the Hamilton energy function of Hindmarsh-Rose neurons was calculated. Wang Chun-Ni et al. [34] extended the Helmholtz theorem [35] in the electromagnetic field theory to dimensionless dynamic systems. Li and Yao [36] analyzed the Hamilton energy of a multivolume attractor chaotic system. Ma et al. [37] designed the Hamilton energy function for three types of attractors and studied the energy modulation of the attractor.

Compared with the general chaotic system, the dynamic behavior of the chaotic system with hidden attractors is more complicated. So, the energy consumption is more, and the Hamilton energy value is lower. In order to obtain the better control effect for the chaotic system with hidden attractors, this paper regards the expected minimum energy consumption as a controller target to control chaotic state of the system. Based on the above analysis, this paper constructs a new dynamic system without equilibrium points and calculates the Hamilton energy function through the Helmholtz theorem, and then an energy feedback controller is designed to control the chaotic system by reducing the energy consumption.

The structure of this article is as follows. The dynamic behavior of the chaotic system with hidden attractors is analyzed in the second part. In the third part, we calculate the Hamiltonian energy function of the improved system and design the system controller. In the fourth part, we do the numerical simulations in order to verify the theoretical results. Conclusions are given in the last part.

\section{Chaotic System with Hidden Attractors}

2.1. Model Building. A chaotic system with special properties can be obtained as follows:

$$
\left\{\begin{array}{l}
\dot{x}=x \\
\dot{y}=-x+a y z+b \cos z, \\
\dot{z}=c-y^{2}
\end{array}\right.
$$

where $x, y$, and $z$ are all state variables and $a, b$, and $c$ are positive real numbers. So, equation (1) is a system with hidden attractors. When $a=15, b=0.01$, and $c=1$, the phase trajectories of system (1) are shown in Figure 1.

2.2. Dynamic Analysis. For system (1), the divergence [38] is as follows:

$$
\nabla V=\frac{\partial \dot{x}}{\partial x}+\frac{\partial \dot{y}}{\partial y}+\frac{\partial \dot{z}}{\partial z}=1+a z
$$

When $-1-a z<0$, system (1) is dissipative. It converges in exponential form $(\mathrm{d} v / \mathrm{d} t)=e^{-(-1-a z) t}$, which means that a volume element with an initial volume $V(0)$ converges to a volume element $V(0) e^{-(-1-a z) t}$ at time $t$.
Therefore, when $t \rightarrow+\infty$, each small volume element including the trajectories of the system converges to zero at an exponential rate $-1-a z$. At this time, the trajectories of system (1) will eventually be limited to a set of limit points. Its asymptotic dynamical behavior will be fixed on an attractor. As the system parameters are changed, different states will appear. To set the initial condition as $(0.1,0.1,0.1)$, the largest Lyapunov exponent is shown in Figures 2(a) and 2(b) as the parameters $a$ and $b$ are changed, respectively. The Lyapunov exponent of system (1) $\lambda_{i}(i=1,2,3)$ is shown in Figure 2(c). The bifurcation diagram of system (1) is shown in Figure 3. From Figures 2 and 3, we can find that, as $\lambda_{1}=0, \lambda_{2}<0$, and $\lambda_{3}<0$, system (1) is in the periodic state. When $\lambda_{1}>0$, $\lambda_{2}=0$, and $\lambda_{3}<0$, system (1) is in the chaotic state.

\section{Hamilton Energy Control}

3.1. Hamilton Energy Theory. Helmholtz theorem decomposes any electromagnetic field into the superposition of the gradient field and vortex field, and the field equation satisfies

$$
F(\vec{r})=F_{d}(\vec{r})+F_{c}(\vec{r})=-\nabla \phi(\vec{r})+\nabla \times A(\vec{r}),
$$

where $F_{d}(\vec{r})$ is the gradient field, $F_{c}(\vec{r})$ is the vortex field, $-\nabla \phi(\vec{r})$ is the negative gradient, $\nabla$ is a vector differential operator, and $A(\vec{r})$ is the vector function. Equation (3) is expressed as any field, which can be decomposed into the negative gradient and the curl. It can be converted into the following equation:

$$
\dot{X}=\frac{\mathrm{d} X}{\mathrm{~d} t}=F_{c}(\vec{r})+F_{d}(\vec{r}),
$$

where $X=\left\{x_{1}, x_{2}, x_{3}, \ldots, x_{n}\right\}$ is a system variable. Therefore, generalized dynamic systems can be used to discuss the Hamilton energy [34] solution of general continuous dynamic systems in this paper. Based on the mean-field theory, the variable evolution of the $n$-dimensional dynamic system can be represented by the dynamic equation system of $n$ variables:

$$
\begin{aligned}
\left(\begin{array}{c}
\dot{x}_{1} \\
\dot{x}_{2} \\
\vdots \\
\dot{x}_{n}
\end{array}\right) & =\left(\begin{array}{cccc}
\frac{\partial F_{1}(X)}{\partial x_{1}} & \frac{\partial F_{1}(X)}{\partial x_{2}} & \cdots & \frac{\partial F_{1}(X)}{\partial x_{n}} \\
\frac{\partial F_{2}(X)}{\partial x_{1}} & \frac{\partial F_{2}(X)}{\partial x_{2}} & \cdots & \frac{\partial F_{2}(X)}{\partial x_{n}} \\
\vdots & \vdots & \vdots & \\
\frac{\partial F_{n}(X)}{\partial x_{1}} & \frac{\partial F_{n}(X)}{\partial x_{2}} & \cdots & \frac{\partial F_{n}(X)}{\partial x_{n}}
\end{array}\right) \times\left(\begin{array}{c}
x_{1} \\
x_{2} \\
\vdots \\
x_{n}
\end{array}\right) \\
& =F_{c}(X)+F_{d}(X) .
\end{aligned}
$$

Suppose the Hamilton energy function of dimensionless dynamic system (5) is $H$, so the Hamilton energy function $H$ satisfies the following conditions: 

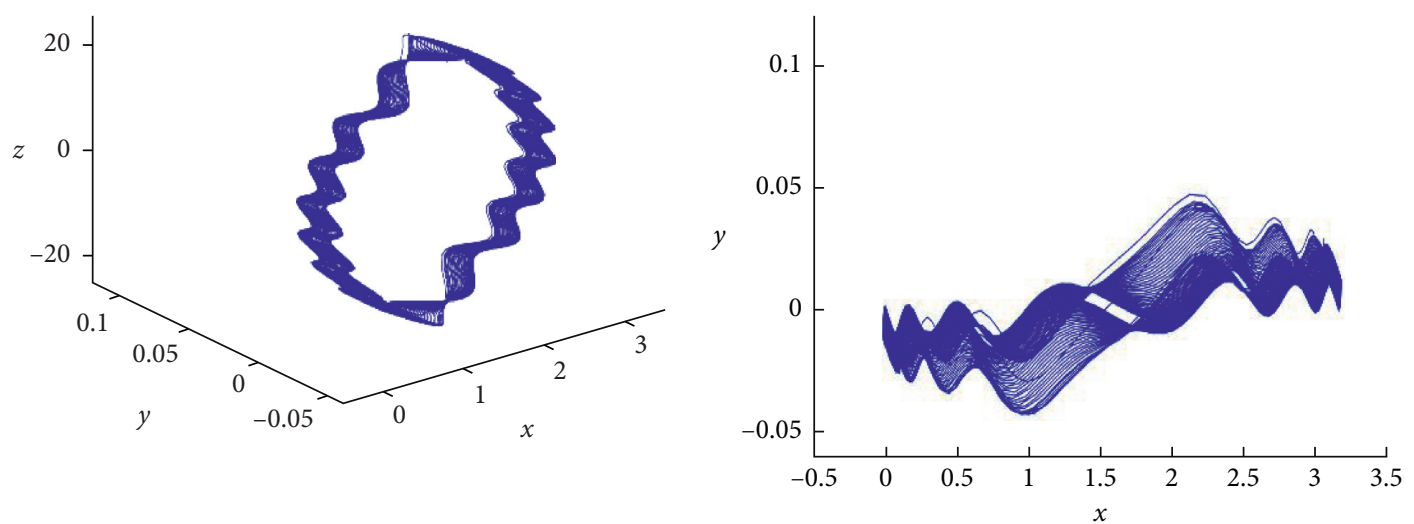

(a)

(b)
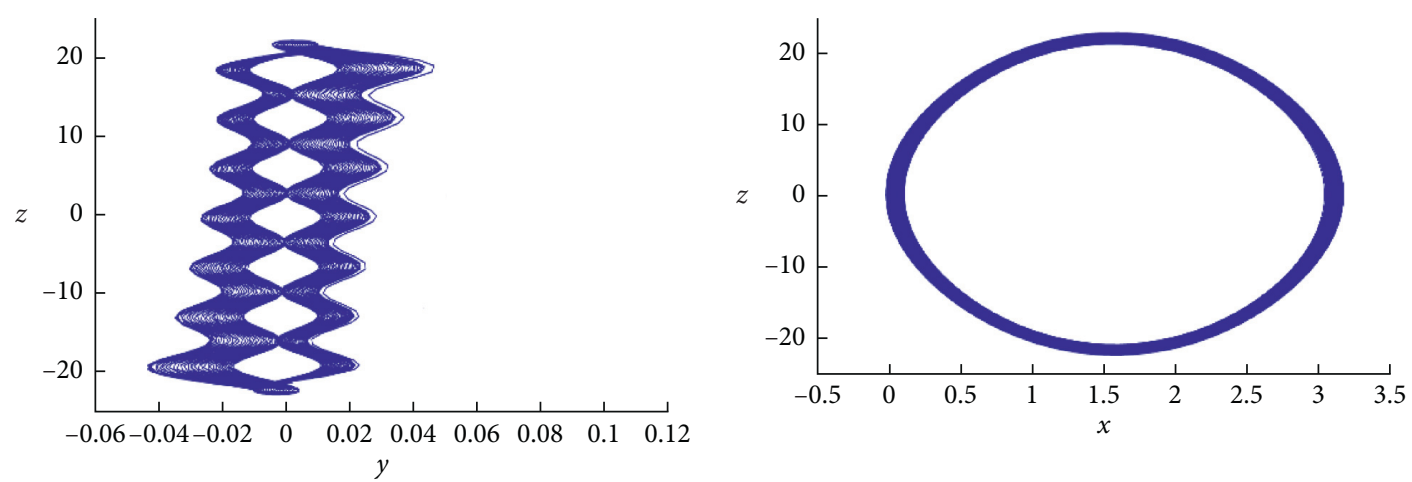

(c)

(d)

Figure 1: Phase trajectories for system (1), $a=15, b=0.01$, and $c=1$.

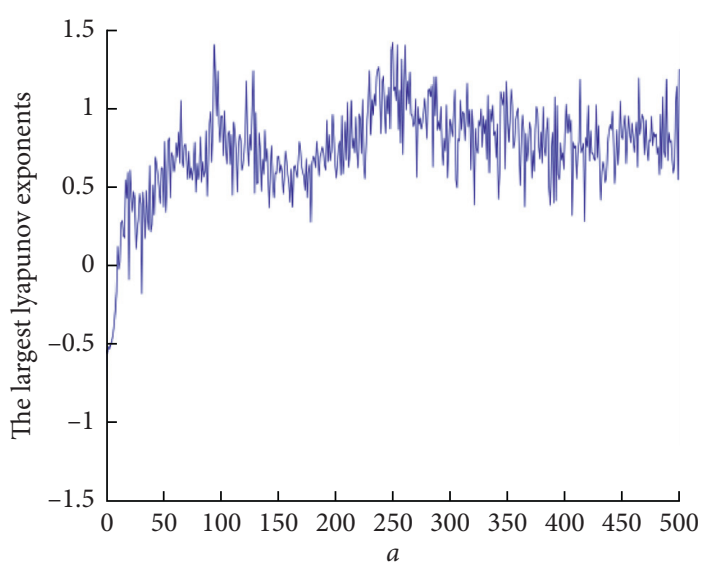

(a)

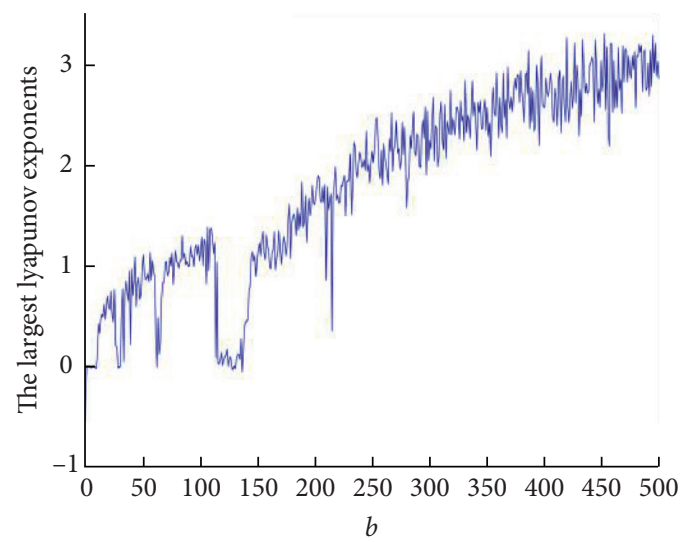

(b)

FIgURE 2: Continued. 


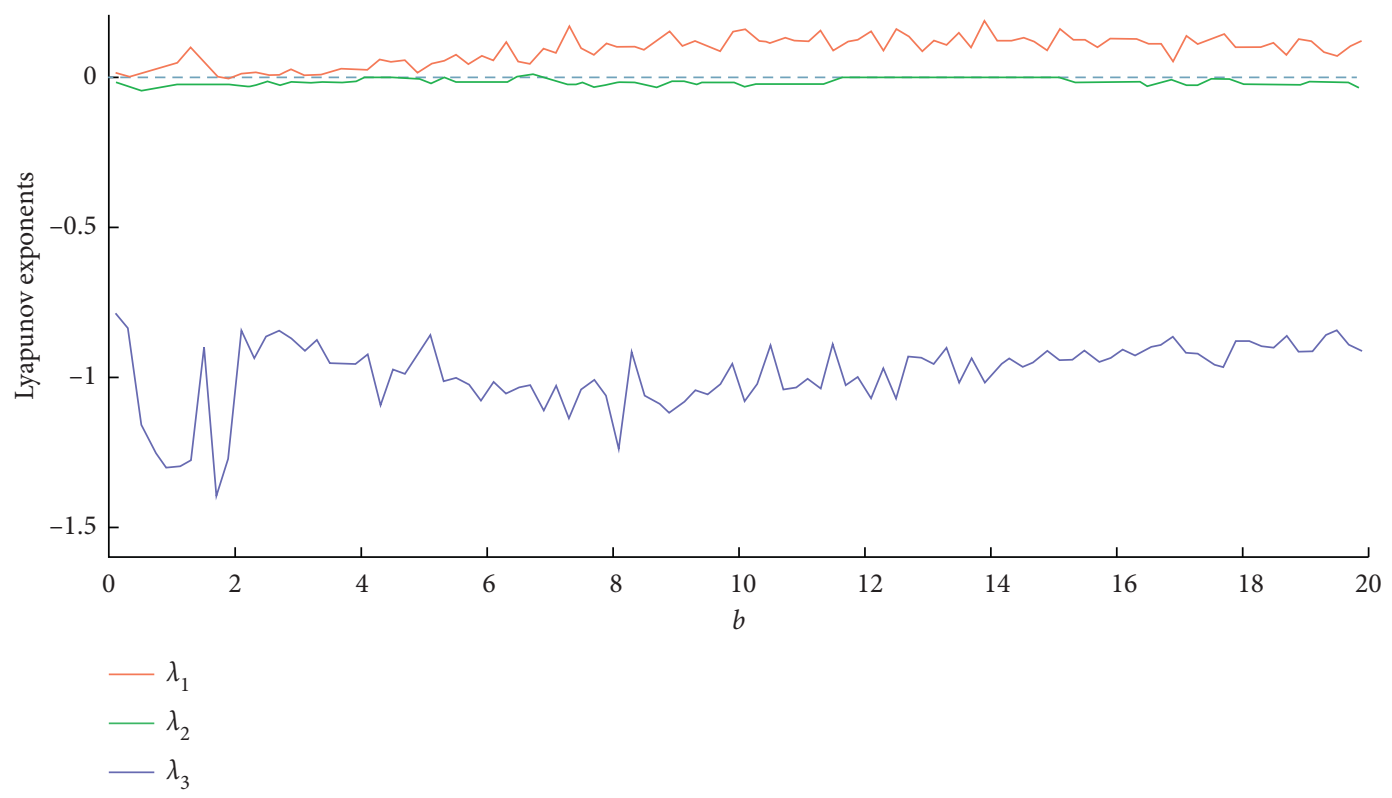

(c)

Figure 2: Lyapunov exponents for system (1). (a) $b=0.01$ and $c=1$. (b) $a=15$ and $c=1$. (c) $a=15, b=0.01$, and $c=1$.

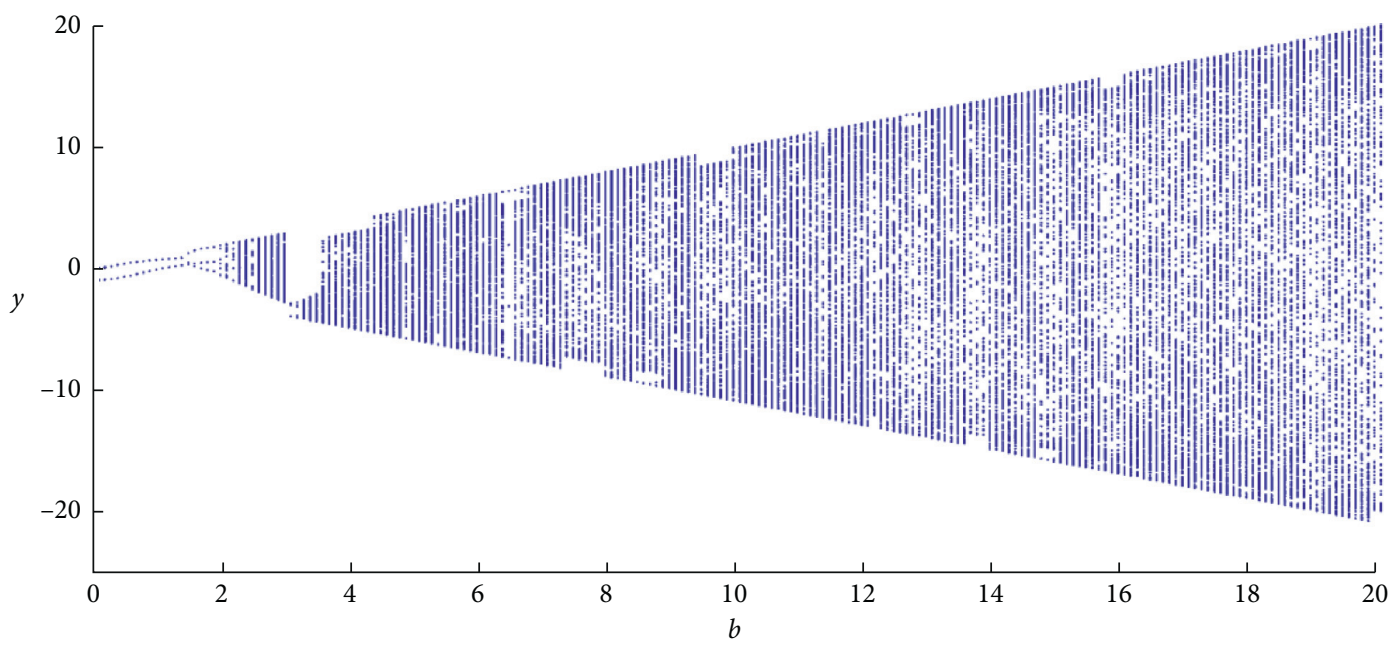

Figure 3: Bifurcation diagram of the variable $y$ for system (1), $a=15$ and $c=1$. 


$$
\begin{aligned}
& \left\{\begin{array}{l}
\nabla H^{T} F_{c}(X)=0, \\
\nabla H^{T} F_{d}(X)=\dot{H}=\frac{\mathrm{d} H}{\mathrm{~d} t} .
\end{array}\right. \\
& F_{c}(X)=\left(\begin{array}{cccc}
0 & \frac{\partial F_{1}(X)}{\partial x_{2}} & \cdots & \frac{\partial F_{1}(X)}{\partial x_{n}} \\
\frac{\partial F_{2}(X)}{\partial x_{1}} & 0 & \cdots & \frac{\partial F_{2}(X)}{\partial x_{n}} \\
\vdots & \vdots & \vdots & \\
\frac{\partial F_{n}(X)}{\partial x_{1}} & \frac{\partial F_{n}(X)}{\partial x_{2}} & \cdots & 0
\end{array}\right) \times\left(\begin{array}{c}
x_{1} \\
x_{2} \\
\vdots \\
x_{n}
\end{array}\right)=J(X) \nabla H, \\
& F_{d}(X)=\left(\begin{array}{cccc}
\frac{\partial F_{1}(X)}{\partial x_{1}} & 0 & \cdots & 0 \\
0 & \frac{\partial F_{2}(X)}{\partial x_{2}} & \cdots & 0 \\
\vdots & \vdots & \vdots & \\
0 & \ldots & \frac{\partial F_{n}(X)}{\partial x_{n}}
\end{array}\right) \times\left(\begin{array}{c}
x_{1} \\
x_{2} \\
\vdots \\
x_{n}
\end{array}\right)=R(X) \nabla H
\end{aligned}
$$

where $F_{d}(X)$ is the gradient field, $F_{c}(X)$ is the vortex field, and $J(X)$ and $R(X)$ are the quasi-symmetric matrix and symmetric matrix, respectively. The direction of $\nabla H$ is vertical to the direction of the vortex field.

The three-variable system is

$$
\left\{\begin{array}{l}
\dot{x}=f_{1}(x, y, z), \\
\dot{y}=f_{2}(x, y, z), \\
\dot{z}=f_{3}(x, y, z) .
\end{array}\right.
$$

The corresponding matrix equation is

$$
\begin{aligned}
\left(\begin{array}{c}
\dot{x} \\
\dot{y} \\
\dot{z}
\end{array}\right)= & \left(\begin{array}{ccc}
\frac{\partial f_{1}(x, y, z)}{\partial x} & \ldots & \ldots \\
\ldots & \frac{\partial f_{2}(x, y, z)}{\partial y} & \ldots \\
\ldots & \ldots & \frac{\partial f_{3}(x, y, z)}{\partial z}
\end{array}\right) \\
& \times\left(\begin{array}{c}
x \\
y \\
z
\end{array}\right)=F_{c}+F_{d} .
\end{aligned}
$$

The dimensionless Hamilton energy function satisfies

$$
\mathrm{d} H=F \cdot \mathrm{d} \vec{r}=F_{x} \mathrm{~d} x+F_{y} \mathrm{~d} y+F_{z} \mathrm{~d} z=\nabla H \cdot \mathrm{d} \vec{r}
$$

$$
=\nabla H^{T} \cdot \mathrm{d} \vec{r}=\left(\frac{\partial H}{\partial x}, \frac{\partial H}{\partial y}, \frac{\partial H}{\partial z}\right)\left(\begin{array}{c}
\mathrm{d} x \\
\mathrm{~d} y \\
\mathrm{~d} z
\end{array}\right),
$$

$$
\dot{H}=\frac{\mathrm{d} H}{\mathrm{~d} t}=\left(\frac{\partial H}{\partial x}, \frac{\partial H}{\partial y}, \frac{\partial H}{\partial z}\right)\left(\begin{array}{c}
\dot{x} \\
\dot{y} \\
\dot{z}
\end{array}\right)=\nabla H^{T} F_{c}+\nabla H^{T} F_{d}
$$

$$
=\nabla H \cdot F_{c}+\nabla H \cdot F_{d}=\nabla H^{T} \cdot F_{d}=\nabla H^{T} F_{d} .
$$

The direction of $\nabla H$ is vertical to the direction of the vortex field (tangent line), and $\nabla H^{T} F_{c}=0$ holds. For any continuous differential dynamic system, the corresponding Hamilton energy function can be obtained based on equation (6).

\subsection{Hamilton Energy Function for System (1). According to} equation (5), the Hamilton energy of system (1) for $F_{c}=$ $\left[\begin{array}{c}0 \\ -x+b \cos z \\ c-y^{2}\end{array}\right]$ and $F_{d}=\left[\begin{array}{c}x \\ a z y \\ 0\end{array}\right]$ can be written as 


$$
(-x+b \cos z) \frac{\partial H}{\partial y}+\left(c-y^{2}\right) \frac{\partial H}{\partial z}=0 .
$$

The general solution of equation (11) is

$$
H=-x z+b \sin z-c y+\frac{1}{3} y^{3} .
$$

Based on the Hamilton theorem, the criterion for the stability of Hamilton energy function $H$ is expressed as equation (5). The first derivative to time of the Hamilton energy function is

$$
\begin{aligned}
\dot{H}= & \frac{\mathrm{d} H}{\mathrm{~d} t} \\
= & -x z-\left(c-y^{2}\right)(-x+a y z+b \cos z) \\
& -(x-b \cos z)\left(c-y^{2}\right) \\
= & -x z-a c y z+a y^{3} z .
\end{aligned}
$$

Substituting equations (12) and (13) into equation (6), we can obtain the following equation:

$$
\begin{aligned}
\nabla H^{T} F_{d} & =\left[-z,-c+y^{2},-x+b \cos z\right]\left[\begin{array}{c}
x \\
a y z \\
0
\end{array}\right] \\
& =-x z-a c y z+a y^{3} z=\dot{H} .
\end{aligned}
$$

As the parameters are selected, $a=15, b=$ 0.01 , and $c=1$, the simulations are shown in Figure 4 . Figure 4(a) is the response of the state variable $y$. Figure 4(b) is the Hamilton energy function for system (1). It can be found from Figure 4 that, as chaos appears, some peak states of the Hamilton energy function and the response will appear at the same time. It is worth noting that the larger value of the response's amplitude appears, and the smaller value of Hamilton energy function appears at the same time. We can know that drastic chaotic oscillation will consume lots of energy. For example, the $\operatorname{dot} Q^{*}$ in Figure 4(a) corresponds to dot $Q$ in Figure 4(b). When $t=139.9$, the amplitude of $Q^{*}$ is equal to 0.6399 , and the Hamilton energy function of $Q$ is equal to -0.0445 . As $t=168.5$, the amplitude decreases to -0.7291 at point $P^{*}$, and more energy function is needed to be given at $H=$ 1.624 at the point $P$.

As the parameters $a, b$, and $c$ are equal to 15,0 , and 1 , respectively, the response and Hamilton energy function are shown in Figures 5(a) and 5(b). From Figure 5, we can know that system (1) is finally stable. When $t=3.207$, the state variable $y$ undergoes the first sharp peak, and then the Hamilton energy function goes through a rapid transformation by decreasing its value. After a period of state evolution, system (1) gradually stabilizes at $t=59.93$. The corresponding Hamilton energy function also approaches zero. This phenomenon can be explained as that system (1) requires a certain amount of energy to maintain a steady state.
As we choose parameters $a=10, b=0.01$, and $c=1$, the response and Hamilton energy function are shown in Figures 6(a) and 6(b), respectively. From Figure 6, we can find a brief chaotic process. At the critical moment of state transition, it enters into a period-like motion state at $t=120$ with more energy consumption. From the above analysis, we can see that the more complex the behavior of the system is, the more the consumption of the energy is. At the same time, the Hamilton energy function value is lower.

It can be seen from equation (13) that Hamilton energy depends on the variables and parameters of the system. The evolution of different states for the system has a great influence on the Hamilton energy function. On the contrary, the change of the Hamilton energy function will also affect the behavior of the chaotic system. Therefore, Hamilton energy control can be applied to control the dynamic behavior in system (1).

The controlled system describing Hamilton energy control is as follows:

$$
\left\{\begin{array}{l}
\dot{x}=x, \\
\dot{y}=-x+a y z+b \cos z, \\
\dot{z}=c-y^{2}-k H \\
\dot{H}=-x z-a c y z+a y^{3} z,
\end{array}\right.
$$

where the parameter $k$ is the feedback coefficient to control the energy flow and $H$ is the Hamilton energy function of system (1). This method controls the dynamic system by increasing or decreasing the parameter $k$ to explore the state change of system (1).

\section{Numerical Simulations}

4.1. Hamilton Energy Feedback Control Simulation. The parameters $a=15, b=0.02$, and $c=1$, and $k$ is equal to $-0.07,0.04$, and 0.18 , respectively; the time history diagrams for system (15) are shown in Figure 7.

Comparing the results of Figure 7, it can be seen that, by changing $k$, the system (1) can be controlled from a chaotic state to an ideal periodic state. Obviously, the Hamilton energy control method is effective. Contrasting the results of Figures $7(\mathrm{~b})$ and $7(\mathrm{c})$, it can be found that to select the appropriate parameter $k$, we can rapidly and effectively control chaos within a limited time.

4.2. Comparison Simulation. In this subsection, the system parameters are chosen as $a=15, b=0.01$, and $c=1$ and the initial condition as $(0.1,0.1,0.1)$. Linear feedback control is the most common control method for studying chaotic systems. This control method is applied to system (1). Let the controller of the system be $u=\left(u_{1}, u_{2}, u_{3}\right)^{T}$, and add the controller to system (1); then, the control system is as follows:

$$
\left\{\begin{array}{l}
\dot{x}=x+u_{1} \\
\dot{y}=-x+a y z+b \cos z+u_{2} \\
\dot{z}=c-y^{2}+u_{3} .
\end{array}\right.
$$




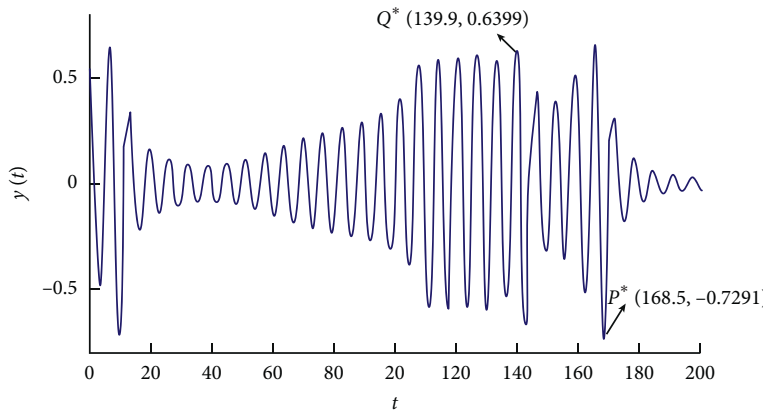

(a)

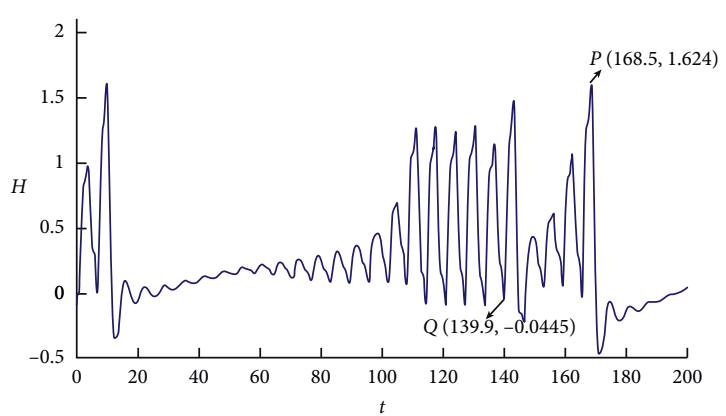

(b)

Figure 4: The time history diagram (a) and Hamilton energy function (b) for system (1), $a=15, b=0.01$, and $c=1$.

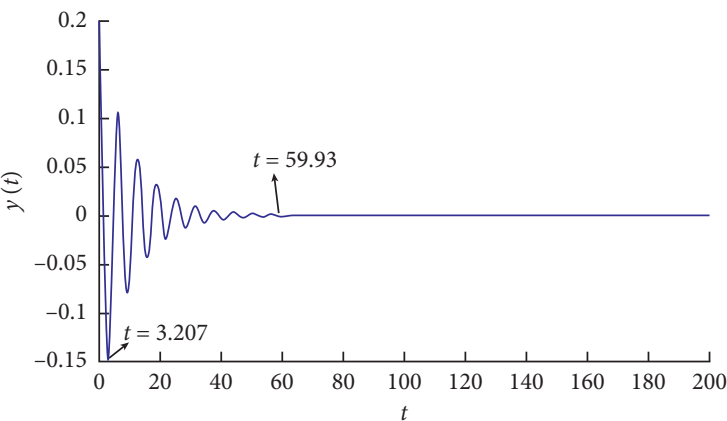

(a)

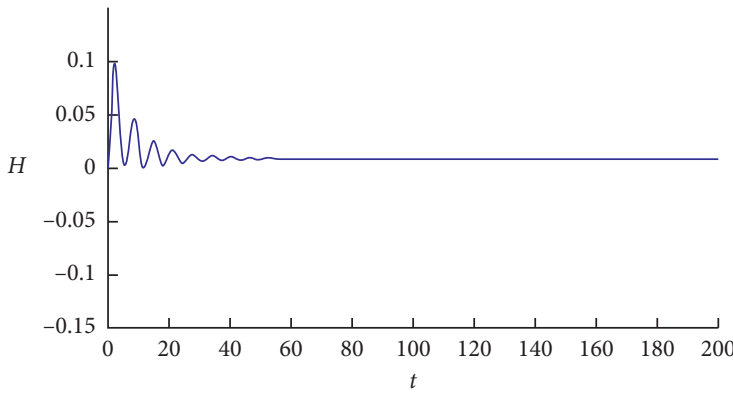

(b)

FIgURe 5: The time history diagram (a) and Hamilton energy function (b) for system (1), $a=15, b=0$, and $c=1$.

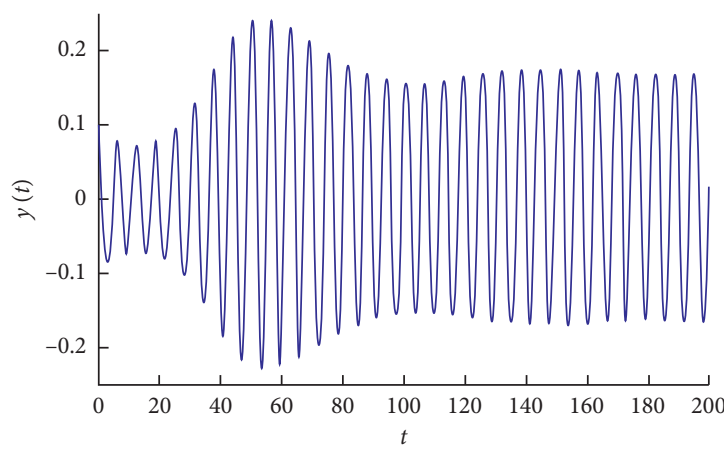

(a)

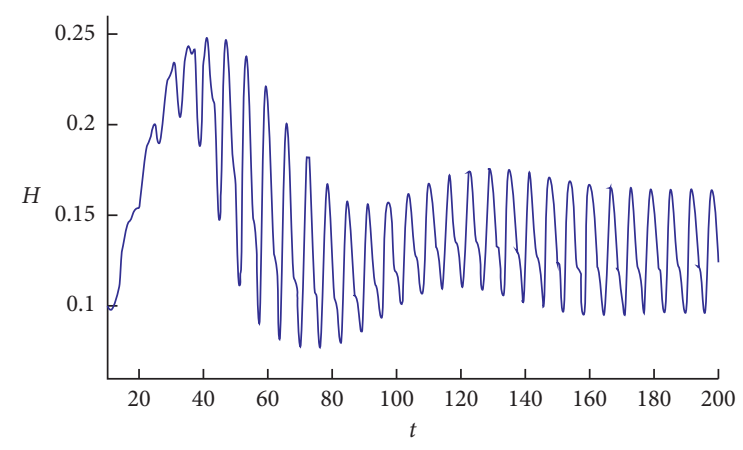

(b)

Figure 6: The time history diagram (a) and Hamilton energy function (b) for system (1), $a=10, b=0.01$, and $c=1$.

According to system (16), the phase trajectories and time history diagram of the system with the linear feedback controller can be obtained in Figure 8. When $u_{1}=0, u_{2}=-0.02$, and $u_{3}=0$, the amplitude of the limit cycle is getting smaller and smaller, which shows that the linear control has a certain impact on system (1).

Figures 9(a) and 9(b) are the phase trajectories and time history diagram of the Hamilton energy control for system (15). The time history diagram for the system Hamilton energy control method and linear feedback control method is shown in Figure 10. It can be seen from Figure 10(a) that, in a certain period of time, the Hamilton energy controller makes the amplitude of the limit cycle smaller and smaller. Figure 10(b) is an enlarged view of Figure 10(a). Especially from Figure 10(b), we can know that the Hamilton energy control method makes the system close to the equilibrium state at $t=27.53$. Above all, the Hamilton energy control system takes less time to reach the equilibrium state than the linear feedback control system. At the same time, controlled system (16) just still goes through the instability state. 


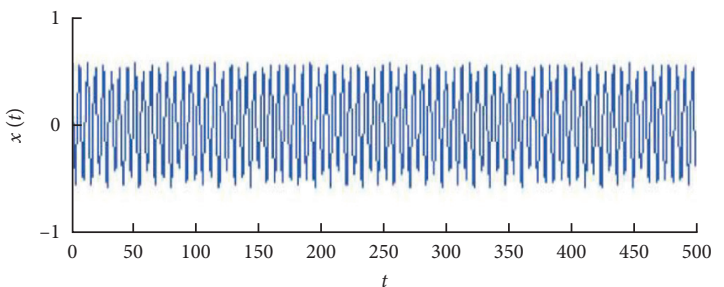

(a)

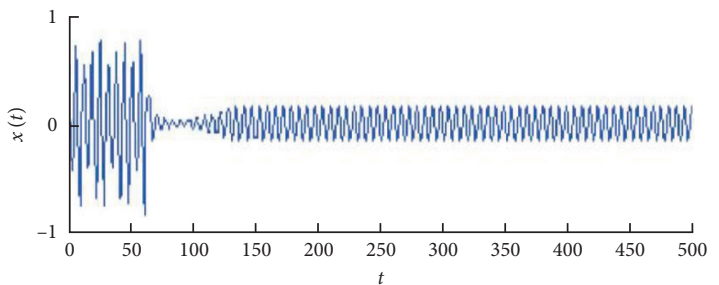

(b)

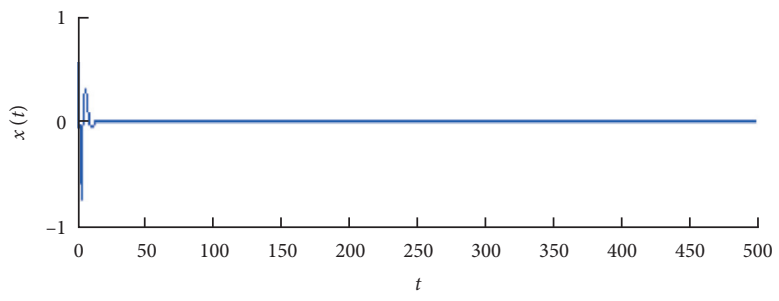

(c)

Figure 7: The time history diagrams for system (15) with parameters as (a) $k=-0.07$, (b) $k=0.04$, and (c) $k=0.18$.

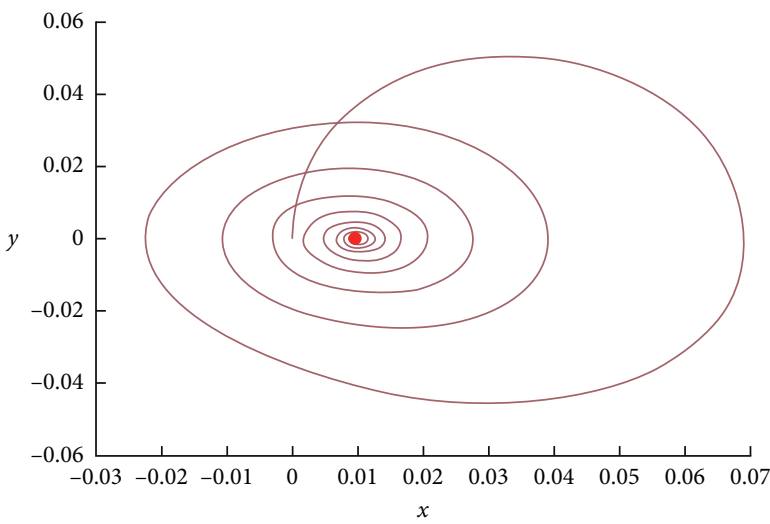

(a)

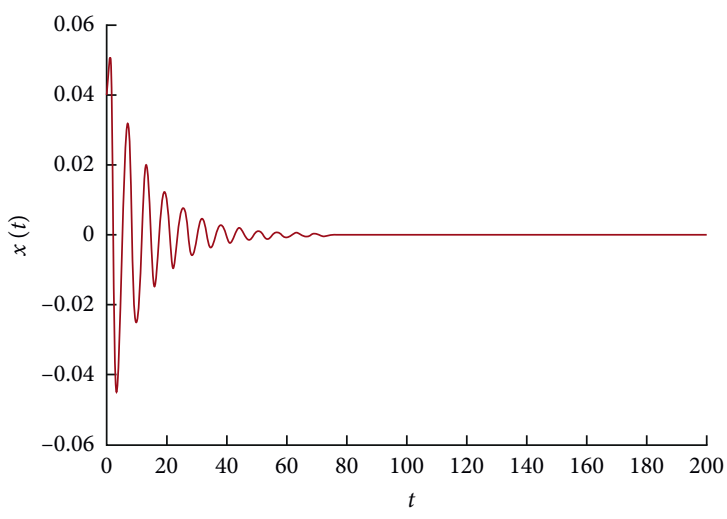

(b)

Figure 8: Phase trajectories (a) and time history diagram (b) for system (16), $a=15, b=0.01$, and $c=1$.

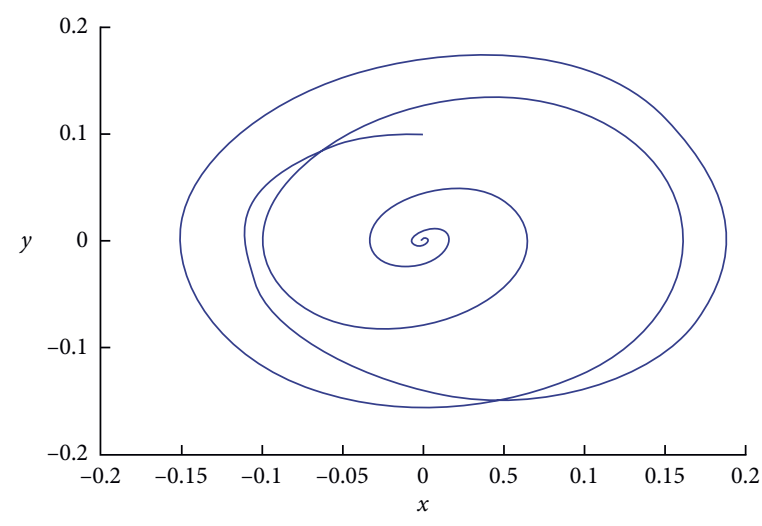

(a)

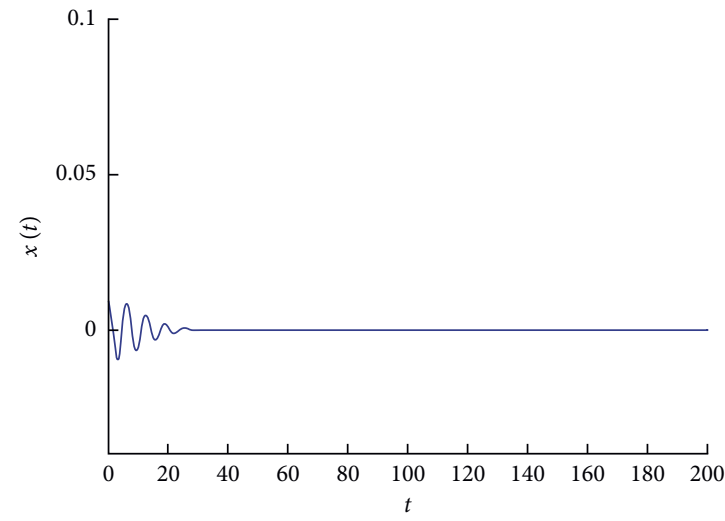

(b)

Figure 9: Phase trajectories (a) and time history diagram (b) for system (15), $a=15, b=0.01, c=1$, and $k=0.02$. 


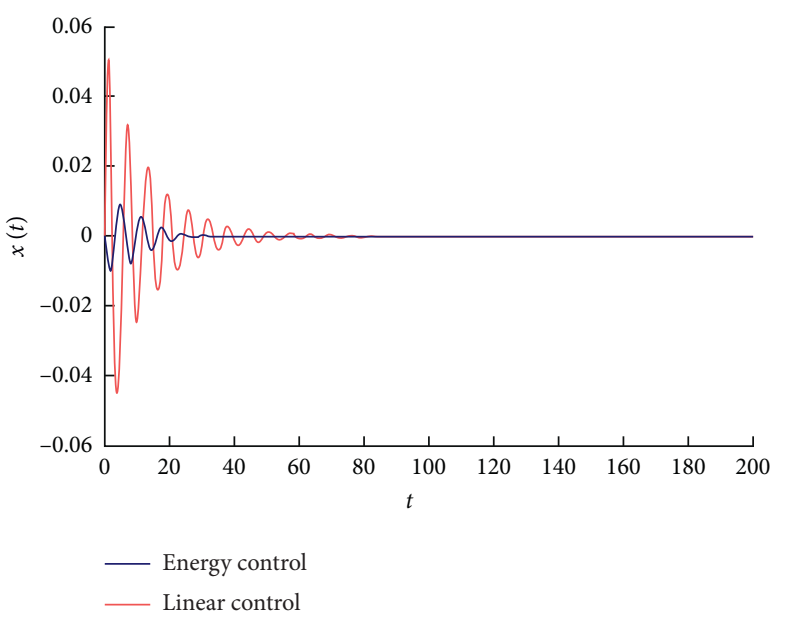

(a)

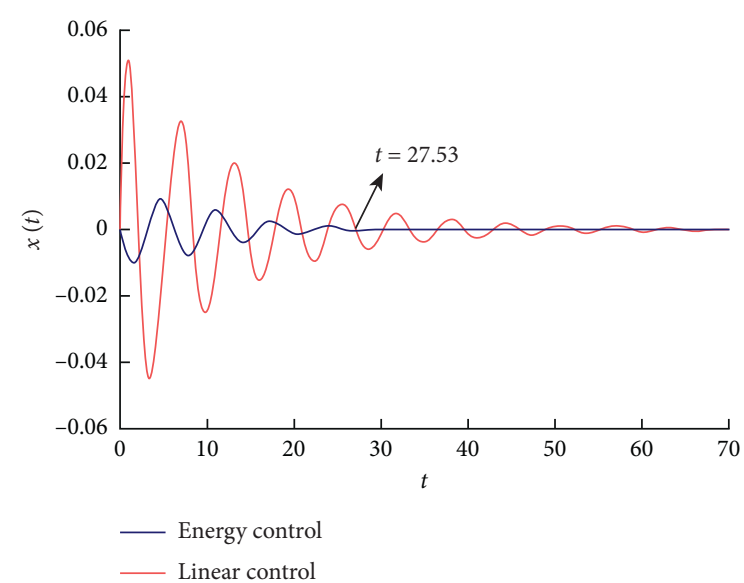

(b)

Figure 10: The time history diagram and partial enlarged diagram for system (15), $k=0.02$, and system (16), $u_{2}=-0.02, a=15, b=0.01$, and $c=1$.

\section{Conclusions}

In this paper, a three-dimensional chaotic system with hidden attractors was designed firstly, and the dynamic behavior of the proposed chaotic system is described by quantitative analysis, such as the bifurcation diagram and largest Lyapunov exponent. Secondly, we calculated the Hamilton energy function by the Helmholtz theorem and applied the Hamiltonian energy control method to control the chaotic system with the cost of minimum energy. At last, numerical simulations were used to verify theory results. We also found that using Hamilton energy control method to chaotic system with hidden attractors was more effective about using less time to gain the same control effect through comparing with linear feedback control method.

\section{Data Availability}

The data used to support the findings of this study have been deposited in the article named "Calculation of Hamilton energy function of dynamical system by using Helmholtz theorem."

\section{Conflicts of Interest}

The authors declare that there are no conflicts of interest regarding the publication of this paper.

\section{Acknowledgments}

The authors thank the National Natural Science Foundation of China (no. 11772002), Major Special Project of North Minzu University (no. ZDZX201902), Open Project of the Key Laboratory of Intelligent Information and Big Data Processing of Ningxia Province (no. 2019KLBD008), Ningxia Higher Education First-Class Discipline Construction Funding Project (NXYLXK2017B09), and North
Minzu University for Nationalities Postgraduate Innovation Project (no. YCX20101) for the support.

\section{References}

[1] P. L. Christiansen, M. P. Sørensen, and A. C. Scott, Nonlinear Science at the Dawn of the 21st Century, Springer, Berlin, Germany, 2000.

[2] G. A. Leonov and N. V. Kuznetsov, "Hidden attractors in dynamical systems. From hidden oscillations in HilbertKolmogorov, Aizerman, and Kalman problems to hidden chaotic attractor in Chua circuits," International Journal of Bifurcation and Chaos, vol. 23, no. 1, Article ID 1330002, 2013.

[3] D. Dudkowski, S. Jafari, T. Kapitaniak, N. V. Kuznetsov, G. A. Leonov, and A. Prasad, "Hidden attractors in dynamical systems," Physics Reports, vol. 637, pp. 1-50, 2016.

[4] G. C. Zheng, C. X. Liu, and Y. Wang, "Dynamic analysis and finite-time synchronization of a fractional-order chaotic system with hidden attractors," Acta Physica Sinica, vol. 67, no. 5, pp. 43-50, 2018.

[5] H. Bao, B. C. Bao, Y. Lin et al., "Hidden attractors and dynamic characteristics of memristive self-oscillation system," Acta Physica Sinica, vol. 65, no. 18, pp. 219-230, 2016.

[6] S. Jafari, J. C. Sprott, and F. Nazarimehr, "Recent new examples of hidden attractors," The European Physical Journal Special Topics, vol. 224, no. 8, pp. 1469-1476, 2015.

[7] G. A. Leonov and N. V. Kuznetsov, "Hidden attractor in smooth Chua system," Physica D: Nonlinear Phenomena, vol. 18, no. 241, pp. 1482-1486, 2012.

[8] S. Brezetskyi, D. Dudkowski, and T. Kapitaniak, "Rare and hidden attractors in Van der Pol-Duffing oscillators," The European Physical Journal Special Topics, vol. 224, no. 8, pp. 1459-1467, 2015.

[9] G. A. Leonov, N. V. Kuznetsov, and M. A. Kiselev, "Hidden oscillations in mathematical model of drilling system actuated by induction motor with a wound rotor," Nonlinear Dynamics,vol. 77, no. 1-2, pp. 277-288, 2014.

[10] G. A. Leonov, N. V. Kuznetsov, and T. N. Mokaev, "Hidden attractor and homoclinic orbit in Lorenz-like system describing convective fluid motion in rotating cavity," 
Communications in Nonlinear Science \& Numerical Simulation, vol. 28, no. 1, pp. 166-174, 2015.

[11] Z. T. Husumbaliyev and E. Mosekilde, "Multistability and hidden attractors in a multilevel DC/DC converter," Mathematics \& Computers in Simulation, vol. 109, pp. 32-45, 2015.

[12] N. Boers, A. Rheinwalt, and B. Bookhagen, "The South American rainfall dipole: a complex network analysis of extreme events," Geophysical Research Letters, vol. 41, no. 20, pp. 7397-7405, 2015.

[13] S. Jafari, A. Ahmadi, A. J. M. Khalaf, H. R. Abdolmohammadi, V.-T. Pham, and F. E. Alsaadi, "A new hidden chaotic attractor with extreme multi-stability," AEU-International Journal of Electronics and Communications, vol. 89, pp. 131135, 2018.

[14] P. R. Sharma, M. D. Shrimali, A. Prasad, N. V. Kuznetsov, and G. A. Leonov, "Control of multistability in hidden attractors," The European Physical Journal Special Topics, vol. 224, no. 8, pp. 1485-1491, 2015.

[15] Y. Feng and Z. Wei, "Delayed feedback control and bifurcation analysis of the generalized Sprott B system with hidden attractors," The European Physical Journal Special Topics, vol. 224, no. 8, pp. 1619-1636, 2015.

[16] S. Vaidyanathan, "A memristor-based hyperchaotic system with hidden attractor and its sliding mode control," Studies in Computational Intelligence, vol. 709, pp. 343-369, 2017.

[17] Z. Wei, A. Akgul, U. E. Kocamaz, I. Moroz, and W. Zhang, "Control, electronic circuit application and fractional-order analysis of hidden chaotic attractors in the self-exciting homopolar disc dynamo," Chaos, Solitons \& Fractals, vol. 111, pp. 157-168, 2018.

[18] Q. Lai, B. Norouzi, and F. Liu, "Dynamic analysis, circuit realization, control design and image encryption application of an extended Lü system with coexisting attractors," Chaos, Solitons \& Fractals, vol. 114, pp. 230-245, 2018.

[19] Q. Lai, Z. Wan, and P. D. Fotsin, "Coexisting attractors, circuit implementation and synchronization control of a new chaotic system evolved from the simplest memristor chaotic circuit," Communications in Nonlinear Science and Numerical Simulation, vol. 89, Article ID 105341, 2020.

[20] Q. Lai, Z. Q. Wan, L. K. Kengne et al., "Two-Memristor-Based chaotic system with infinite coexisting attractors," IEEE Transactions on Circuits and Systems II-Express Briefs, vol. 68, pp. 2197-2201, 2020.

[21] Q. Lai, "A unified chaotic system with various coexisting attractors," International Journal of Bifurcation \& Chaos, 2021.

[22] Q. Lai, Z. Wan, and P. D. Kamdem Kuate, "Modelling and circuit realisation of a new no-equilibrium chaotic system with hidden attractor and coexisting attractors," Electronics Letters, vol. 56, no. 20, pp. 1044-1046, 2020.

[23] F. D. Marius and F. Michal, "Hidden chaotic attractors and chaos suppression in an impulsive discrete economical supply and demand dynamical system," Communications in Nonlinear Science and Numerical Simulation, vol. 74, pp. 1-13, 2019.

[24] Q. Wu, Q. Hong, X. Liu, X. Wang, and Z. Zeng, “A novel amplitude control method for constructing nested hidden multi-butterfly and multiscroll chaotic attractors," Chaos, Solitons \& Fractals, vol. 134, Article ID 109727, 2020.

[25] N. P. Mohanty, R. Dey, and B. K. Roy, "Switching synchronisation of a 3-D multi-state-time-delay chaotic system including externally added memristor with hidden attractors and multi-scroll via sliding mode control," European Physical Journal Special Topics, vol. 229, no. 6-7, pp. 1231-1244, 2020.
[26] V. I. Arnold, "Mathematical methods of classical mechanics," Advances in Mathematics, vol. 49, no. 1, p. 106, 1983.

[27] J. B. Li, Generalized Hamiltonian Systems Theory and its Applications, Science Press, Beijing, China, 1994.

[28] H. Sira-Ramírez and C. Cruz-Hernández, "Synchronization of chaotic systems: a generalized Hamiltonian systems approach," International Journal of Bifurcation and Chaos, vol. 11, no. 5, pp. 1381-1395, 2001.

[29] C. Sarasola, F. J. Torrealdea, A. D'Anjou, A. Moujahid, and M. Graña, "Energy balance in feedback synchronization of chaotic systems," Physical Review E, vol. 69, no. 1, Article ID 011606, 2004.

[30] F. J. Torrealdea, A. d'Anjou, M. Graña, and C. Sarasola, "Energy aspects of the synchronization of model neurons," Physical Review E, vol. 74, no. 1, Article ID 011905, 2006.

[31] F. J. Torrealdea, C. Sarasola, and A. d'Anjou, "Energy consumption and information transmission in model neurons," Chaos, Solitons \& Fractals, vol. 40, no. 1, pp. 60-68, 2009.

[32] A. Moujahid, A. D'Anjou, and F. J. Torrealdea, "Energy cost reduction in the synchronization of a pair of nonidentical coupled Hindmarsh-Rose neurons," Physics Reports, vol. 22, no. 16, pp. 657-664, 2012.

[33] X.-L. Song, W.-Y. Jin, and J. Ma, "Energy dependence on the electric activities of a neuron," Chinese Physics B, vol. 24, no. 12, Article ID 128710, 2015.

[34] C. N. Wang Chun-Ni, Y. N. Wang Ya, and J. Ma Jun, "Calculation of Hamilton energy function of dynamical system by using Helmholtz theorem," Acta Physica Sinica, vol. 65, no. 24, Article ID 240501, 2016.

[35] D. H. Kobe, "Generalization of Coulomb's law to Maxwell's equations using special relativity," American Journal of Physics, vol. 54, no. 7, pp. 631-636, 1986.

[36] F. Li and C. Yao, "The infinite-scroll attractor and energy transition in chaotic circuit," Nonlinear Dynamics, vol. 84, no. 4, pp. 2305-2315, 2016.

[37] J. Ma, F. Wu, W. Jin, P Zhou, and T Hayat, "Calculation of Hamilton energy and control of dynamical systems with different types of attractors," Chaos, vol. 27, pp. 053108053495, 2017.

[38] S. Wu and X. J. Yang, "Multi-sensor control based on CauchySchwarz divergence," Computer Technology and Development, vol. 278, no. 6, pp. 166-172, 2020. 